production of isotopes in uranium piles and cyclotrons, and the reader is given a clear description of the requirements for these processes. More stress might have been laid, however, on the high specific activities produced by the Szilard-Chalmers effect.

The chapter on the detection and measurement of the radiations includes sections on units, Geiger counters of various types, proportional and scintillation counters. Absolute and relative counting are both considered and a section is devoted to the statistics of counting experiments. Adequate reference is made, for example, to the comparison of radioactive sources under exactly similar conditions. It is a pity that occasionally one is left in doubt about the exact scope and limitation of a few of the counting methods. The addition of more numerical examples, here and elsewhere, would add greatly to the value of the book.

The authors deseribe the gross effect of the radiations, the production of ionization and the physical and chemical consequences. Dose units and dose rates from various sources, ionization chambers, electrometers, and calorimeters are discussed. The chemical aspects are dealt with very briefly.

The treatment of the applications of radioactivity lays stress on the principles and validity of the methods. The radioactive isotopes and their compounds can be used as markers, tracers and sources of radiation, and illustrative examples are widely chosen from physics, chemistry, biology, medicine and industry : sound advice is offered on the design of experiments. The book finishes with a description of manipulation techniques and hazards, and a useful table of many radioactive isotopes.

The index would be improved by including some of the more common isotopes. Throughout the book, graphs and formulæ have been presented in useful forms, and many references are made to original papers, reviews and books: readers who wish to translate the written word into action will find valuable information in this book, though those who are not physicists might appreciate more information on the characteristics of the ideal electronic equipment to be used in conjunction with radioactive isotopes. This would enable an experimenter to assess the power packs, amplifiers, scalars and other equipment available commercially.

In spite of these minor criticisms, the book can be thoroughly recommended. S. J. THомSON

\section{DISCHARGES IN GASES}

\section{Electrical Breakdown of Gases}

By J. M. Meek and J. D. Craggs. (International Series of Monographs on Physics.) Pp. ix +507. (Oxford : At the Clarendon Press ; London : Oxford University Press, 1953.) 60s. net.

GIR JOHN TOWNSEND advanced his theory of $S$ ionization by collision in 1900 and his theory of the unstable discharge in 1903 (in The Electrician). The brilliant researches he afterwards conducted and described in his 1915 book "Electricity in Gases" gave most readers the impression that the funda. mental theory of discharges was known and that it remained but to apply it. After 1920 this optimism disappeared mainly because the principles of the interactions between electrons, ions, molecules, radiation and solid surfaces were incompletely known and because the range of possible conditions permits a remarkable proliferation of phenomena. The great volume of research undertaken since then provided a vast quantity of observed data, greatly improved experimental methods and valuable deductions within limited fields, but the general theory did not greatly advance. Since the Second World War, it has come to be appreciated that Townsend had the roots of the matter and that, with the aid of contemporary advances in physics, workers on discharges can begin to see the complete tree.

Prof. Meek has for long been one of the foremost contributors to the subject, at first with Dr. T. E. Allibone, then with Prof. L. B. Loeb and now with the school he founded himself at Liverpool, in which Dr. Craggs plays a leading part. The authors of "Electrical Breakdown of Gases" are thus essentially qualified for their task of summarizing knowledge of unstable conduction in gases. Their book is as complete as its five hundred pages and profuse, and often beautiful, illustrations allow. It is highly compressed and its twelve chapters have impressive bibliographies. One can only hint at its scope. The first five chapters give the principles of ionic motion, the mechanisms of ionization and recombination, and the concept of the two Townsend ionization coefficients which together can multiply the ionization into an avalanche and can make the discharge self-maintaining. In the light of these principles the behaviour is discussed of discharges at low pressures, then the corona and the spark at higher pressures, where they are distinct phenomena, and finally the lightning discharge, which is the leading example of the long spark caused by a transient voltage. Chapter 6 essays a quantitative theory of the spark based mainly on the streamer and radial-field hypothesis. Chapter 7 deals with the numerical sparkover voltages in cases of practical importance, including the uniform field, the sphere gap and concentric cylinders. Chapters 8,9 and 11 cover studies ancillary to or somewhat removed from the main analysis, namely, the influence of ionizing radiations, discharges at high frequency and electrode effects. Chapter 10 considers the detailed structure of the actual spark channel as obtained by recent experimental techniques and relates it to theory. The final chapter deals with the important problem of the transitions from the high-resistance glow to the low-resistance arc condition, which may occur in time or in space. Throughout the book reference is made to important engineering and scientific applications at points where the principle discussed is that which determines the application.

The appendix on recent developments might be thought to support a criticism that the authors should have waited to see whether current work is ultimately successful in simplifying some explana. tions, for example, the direct application of Townsend's equations by Prof. Llewellyn Jones. On the other hand, any later simplification would be offset by the discovery of new phenomena and a valuable and necessary book would stay unwritten. Similarly, though some may think the authors take rather an individual point of view on controversial issues, such as the streamer theory, yet they refer to those who disagree with them, so that the reader can examine the literature and decide for himself. If books are to be written by those who are building up the subjects they treat, then such books must necessarily refect the original research of their authors. Prof. Meek and Dr. Craggs have written a book which every physicist or engineer interested in gaseous discharges should not merely read but should always consult.

S. WhITEHEAD 